\title{
Diagnostics of Corrosion Protection Potential for Electric Water Heaters
}

\author{
Valeriy Martynyuk ${ }^{1 *}$, Michat Liss $^{2}$ and Joanna Wilczarska ${ }^{2}$ \\ ${ }^{1}$ Programming and Computer, Telecommunication Systems Faculty in Khmelnytsky National \\ University, Department of Telecommunication and Computer Integrated Technology, 29016, 11, \\ Instytuts'ka str., Khmelnitsky, Ukraine \\ ${ }^{2}$ Faculty of Mechanical Engineering UTP in Bydgoszcz, Department of Vehicle Engineering, Al. \\ Prof. S. Kaliskiego 7, 85-796 Bydgoszcz, Poland
}

\begin{abstract}
The paper represents the mathematical model for diagnostics of the corrosion protection potential for the electric water heaters (EWH). The research objectives are the problem of determining the EWH corrosion protection technical condition during its operation. The EWH corrosion protection diagnostics is based on the mathematical model of the corrosion protection potential for EWH. The model is based on the nonlinear equivalent circuit which is modelling the nonlinear impedance of the corrosion cathodic protection. In the development of the mathematical model the authors carried out experimental measurements of the impedance for the EWH body fragment. The obtained experimental results show that mathematical model includes the fractional integrating elements. By using of the experimental results the expressions of the impedance, the impulse response is obtained. The methodological approach to increasing the reliability of the EWH corrosion protection diagnostics has been proposed, in terms of the multi-parameter diagnostics, by applying the EWH corrosion protection impedance model.
\end{abstract}

\section{Introduction}

Protection of metals from corrosion is one of the most important national economic problems, since losses from corrosion in industry, transport and other sectors of the economy are comparable to the costs of developing the largest industries. Technological and household systems for storing and processing industrial and drinking water are in a number of metal structures with increased labour input in manufacturing. A significant part of the products is manufactured using low alloy carbon steel with an enameled surface.

The use of alloyed stainless steel increases the cost of production, reduces its market quality. In addition, the increased content of chlorine ions in water and aqueous electrolytes reduces the corrosion resistance and can cause local or point destruction of the surface of alloys. In view of this, instruments and assemblies made of stainless steel should also be equipped with corrosion protection [1].

The rate of metal corrosion in water, soil and aqueous solutions depends on the potential $E$ of the material in the environment. When the potential is shifted towards the negative values, the corrosion rate decreases, which is achieved by means of direct electric current applied through the anode system to the metal surface of the protected object.

The material wear rate defined by the standards should be less than $0.01 \mathrm{~mm}$ per year. This wear rate provides the intended service life of the object, at which the influence of any corrosive wear is not manifested.

Electric water heaters are also called boilers. Their internal volume is filled with water, which is heated in the process of their work. The inner working surface of the electric water

* Corresponding author: martynyuk.valeriy@gmail.com 
heater is in constant contact with the electrolyte, since ions of different metals are in the water. Thus, it can be concluded that the inner working surface of an electric water heater is exposed to electrochemical corrosion.

The cathodic protection of the metal surface, due to its manufacturability and the possibility of application in a wide range of environmental parameters, is by far the most common way of corrosion protection [2]. The cathodic protection is based on a shift of the metal potential towards negative values, while the rate of the anode reaction decreases in case of cathodic polarization, which causes a decrease in the corrosion rate.

Electric water heaters are one of the most common types of enameled household heating equipment [3]. According to EWH type, they are classified into: flow, storage (capacitive) and flow-storage. The design and operation of all capacitive EWH are almost the same as shown in fig. 1.

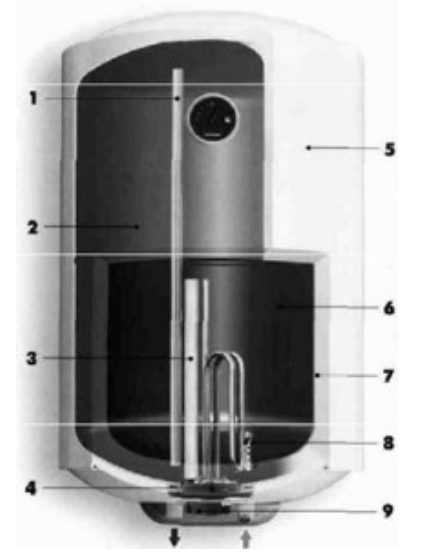

Fig. 1. EWH Design: 1- hot water outlet pipe; 2 - glass-enamel coated inner steel tank; 3 magnesium anode; 4 - steel flange on which heating element, magnesium anode and thermostat are mounted; 5 - polymer-coated external steel tank; 6 - glass-enamel coating; 7 - polyurethane foam insulation; 8 - cold water supply pipe; 9 - thermostat.

EWH consists of inner and external steel tanks, the space between which is filled with insulating materials. Water heaters, a thermostat, and a safety valve are built into the inner tank.

The inner tank is made of low-carbon $1.5-2.0 \mathrm{~mm}$ sheet steel, and then coated with protective glass enamel. Upon contact of the inner steel tank with hot water in places of damage to the glass-enamel protective coating, intense corrosion of the metal surface occurs, which leads to destruction of the product.

The main reason for shortening the service life of a capacitive EWH leading to its failure is corrosion of its inner steel tank. Glass-enamel coating does not provide an EWH with complete protection from corrosion, because during its operation microcracks and other small defects can be formed due to cyclic thermal loads.

To avoid corrosion of defective coating areas of the EWH inner steel tank (not protected with glass-enamel coating), the cathodic protection method is used.

The main criteria of cathodic protection are the corrosion protection potential, as well as methods and devices for its diagnostics. Protective potential is the potential at which the metal dissolution rate takes an extremely low value, acceptable for these operating conditions.

\section{Reliability of the EWH protection potential diagnostics}

Reliability of diagnostics is the degree of objective conformity of the result of diagnostics to the actual technical condition of the corrosion protection potential, which is estimated by 
the probability of making the correct decision about the condition of the corrosion protection potential. The components of the general reliability of diagnostics are methodological and instrumental reliabilities of diagnostics [4]:

$$
D_{G}=D_{M} \cdot D_{I}
$$

where: $D_{M}$ is methodological reliability of diagnostics; $D_{I}$ is instrumental reliability of diagnostics.

Methodological reliability of diagnostics is calculated by the following formula:

$$
D_{M}=\frac{N_{1}}{N}
$$

where: $\mathrm{N}_{1}$ is the number of exponents which characterize the technical condition of the supercapacitor and are included into the mathematical model of diagnostics;

$\mathrm{N}$ is total number of exponents which characterize the diagnostics of the EWH protection potential.

Instrumental reliability of diagnostics is defined by the probabilities of errors of the first and second kind respectively $\alpha$ i $\beta[5]$ :

$$
D_{I}=1-\alpha-\beta
$$

where: $\alpha$ is probability of errors of the first kind; $\beta$ is probability of errors of the second kind.

The overall reliability of diagnostics of the corrosion protection potential of EWH bodies $D_{G}$ may be increased by increasing its two components: methodological reliability of diagnostics $D_{M}$ and instrumental reliability of diagnostics $D_{I}$.

Increase of the methodological reliability of diagnostics of the corrosion protection potential of EWH bodies $D_{M}$ is carried out in the process of diagnostics by determining a large number of parameters that affect the diagnostic object.

Increase of the instrumental reliability of diagnostics of the corrosion protection potential of EWH bodies $D_{I}$ is carried out by reducing the measurement error of controlled parameters.

\section{Process of the EWH corrosion protection potential diagnostics}

In general, the process of the EWH corrosion protection potential diagnostics comprises three main stages as shown in fig. 2.

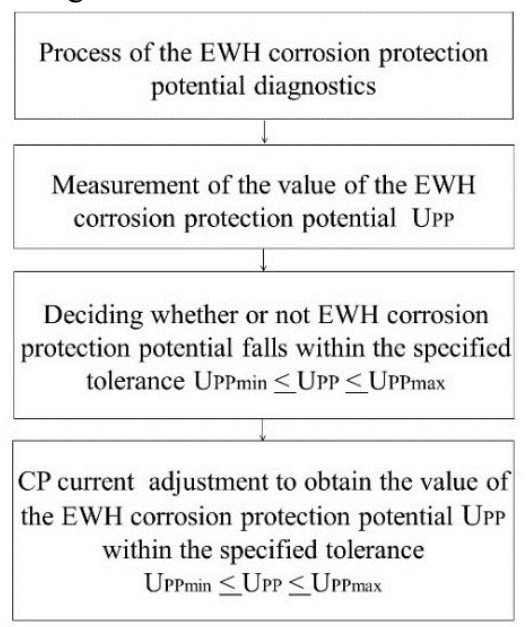

Fig. 2. The stages of the EWH corrosion protection potential diagnostics. 
1. Measurement of the value of the EWH corrosion protection potential $U_{P P}$.

2. Deciding whether or not EWH corrosion protection potential falls within the specified tolerance $U_{P P \min } \leq U_{P P} \leq U_{P P \max }$. According to German standards DIN 509272013 the EWH corrosion protection is provided at the maximum value $U_{P P \max }=-540 \mathrm{mV}$. The minimum value of the protection potential $U_{P P \min }=-1,1 \mathrm{~V}$ is limited by the voltage of water decomposition releasing hydrogen and oxygen and generating explosive gas. Generation of explosive gas is not permitted for safety reasons.

3. CP current $I_{C P}$ adjustment to obtain the value of the EWH corrosion protection potential $U_{P P}$ within the specified tolerance $U_{P P \text { min }} \leq U_{P P} \leq U_{P P \max }$.

At the interface between the metal and the electrolyte solution, an electric double layer (EDL) appears [6-8]. Figure 3 presents an equivalent circuit of the protected from corrosion object (cathode) and the anode, located in a conductive medium (water).

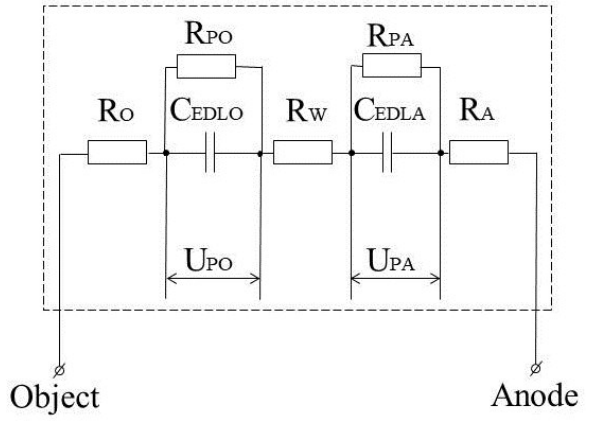

Fig. 3. The equivalent circuit of the object (cathode) and the anode, located in the conductive medium.

On the surface of the protected object (cathode), as well as on the surface of the anode, two EDL appear, which in fig. 3 are shown as two capacitors. Also, fig. 3 represents the active resistance $R_{O}$ and polarization resistance $R_{P O}$ of the protected object (cathode), the active resistance $R_{A}$ and polarization resistance $R_{P A}$ of the anode, which are connected through the active resistance of the conductive medium (water) $R_{W}$.

While developing an equivalent circuit (fig. 3), experimental measurements of the impedance of an EWH body fragment (cathode, fig. 4 a) in the electrolyte solution (tap water, fig. 4 b) and anode (fig. 4 c) were carried out.

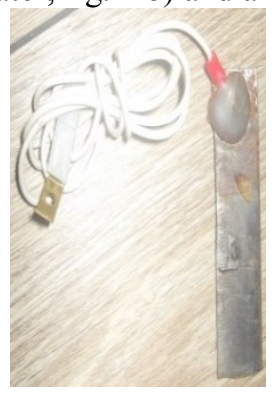

a)

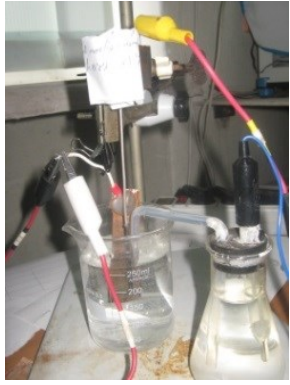

b)

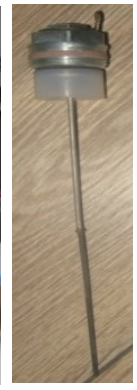

c)

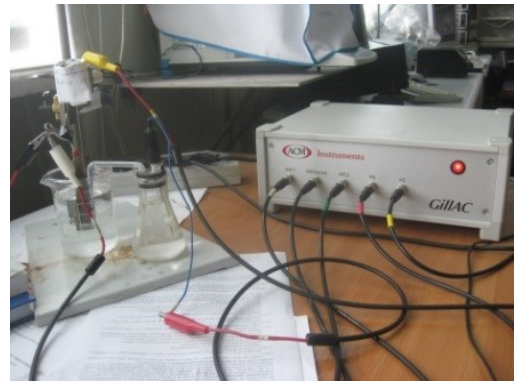

d)

Fig. 4. Pictures of the experimental setup components: a) an EWH body fragment (cathode); b) electrolyte solution (tap water); c) anode; d) experimental setup. 
The measurements were carried out within the Department of Physicochemical Methods of Metal Corrosion Protection at Karpenko Physico-Mechanical Institute of the NAS of Ukraine (Lviv) using a GillAC impedance meter produced by ACM Instruments (Great Britain). A picture of the experimental setup is shown in fig. $4 \mathrm{~d}$.

The adequacy of the equivalent circuit (fig. 3) is confirmed by the coincidence of the theoretical and experimental Nyquist diagrams, which are shown in fig. 5.

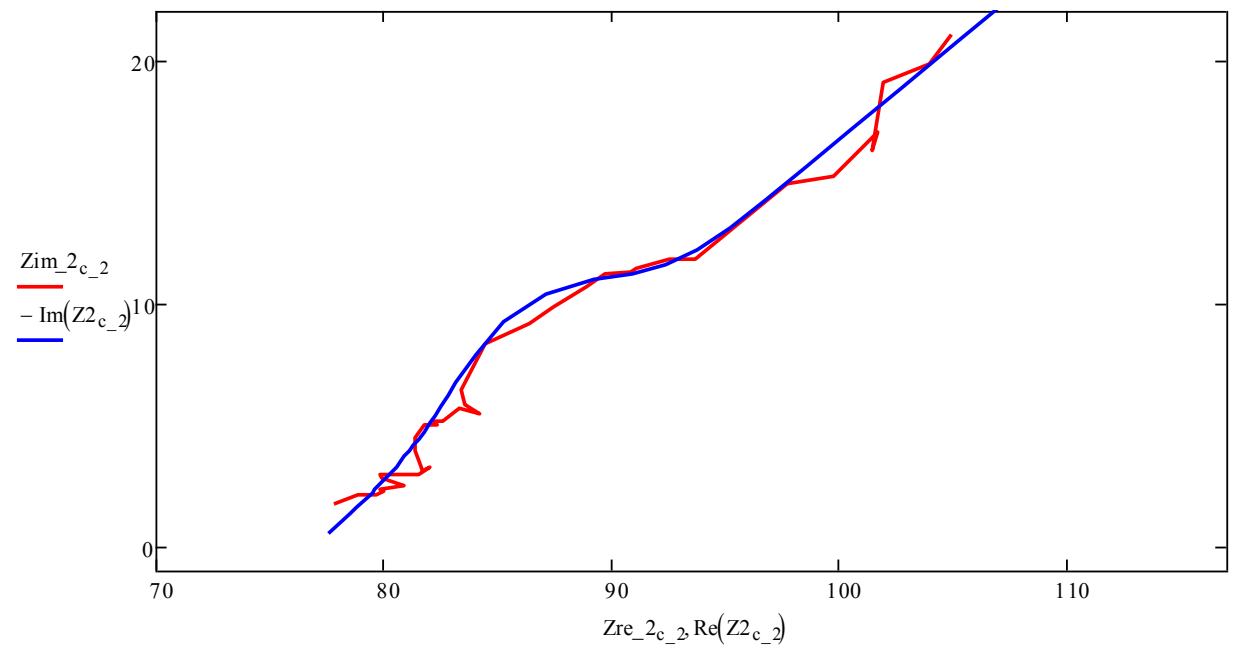

Fig. 5. Theoretical (blue curve) and experimental (red curve) Nyquist diagrams of the equivalent circuit of the EWH and anode, located in the conductive medium.

\section{Mathematical model of EWH corrosion protection potential diagnostics}

Approximation of the experimental Nyquist diagram by the theoretical Nyquist diagram is performed by the impedance function (4) in the frequency range from $F_{\min }=10 \mathrm{mHz}$ to Fmax $=187 \mathrm{~Hz}$ applying the least squares method (LSM).

$$
\begin{aligned}
& Z(j \omega)=R_{O}+\frac{R_{P O}}{1+(j \omega)^{\alpha} C_{E D L O} R_{P O}}+R_{W}+\frac{R_{P A}}{1+(j \omega)^{\beta} C_{E D L A} R_{P A}}+R_{A}= \\
& =R_{E Q}+\frac{R_{P O}}{1+(j \omega)^{\alpha} C_{E D L O} R_{P O}}+\frac{R_{P A}}{1+(j \omega)^{\beta} C_{E D L A} R_{P A}}
\end{aligned}
$$

where: $R_{E Q}=R_{O}+R_{W}+R_{A}$ is equivalent active resistance.

The approximation resulted in the following values of the parameters of the equivalent circuit of the EWH and anode, located in a conductive medium (water) are: $\mathrm{R}_{\mathrm{EQ}}=77 \mathrm{Ohm}$, $\mathrm{R}_{\mathrm{PO}}=2.9 \mathrm{Ohm}, \mathrm{C}_{\mathrm{EDLO}}=0.07 \mathrm{~F}, \alpha=1.4, \mathrm{R}_{\mathrm{PA}}=1 \mathrm{kOhm}, \mathrm{C}_{\mathrm{EDLA}}=0.05 \mathrm{~F} ; \beta=0.45$.

To find the impulse response of the equivalent circuit of the EWH-water-anode system (fig. 3), let us put down the impedance function (4) of the circuit in the operator form, replacing $j \omega$ by $s$ :

$$
Z(s)=R_{E Q}+\frac{R_{C P}}{1+p^{\alpha} C_{E D L C} R_{C P}}+\frac{R_{A P}}{1+p^{\beta} C_{E D L A} R_{A P}}
$$

Then the impulse response of the equivalent circuit of the EWH-water-anode system can be determined as the Laplace transform of the impedance function (5) of the circuit in the operator form:

$$
z(t)=L[Z(s)]
$$




$$
z(t)=R_{E Q} \delta(t)+\frac{t^{\alpha-1}}{C_{E D L C}} E_{\alpha, \alpha}\left[-\frac{t^{\alpha}}{R_{C P} C_{E D L C}}\right]+\frac{t^{\beta-1}}{C_{E D L A}} E_{\alpha, \alpha}\left[-\frac{t^{\beta}}{R_{P A} C_{E D L A}}\right]
$$

where: $E_{\alpha, \alpha}[\bullet]$ is Mittag-Leffler function.

The impulse response graph of the equivalent circuit of the EWH-water-anode system calculated using MATLAB R2015b is shown in fig. 6. To implement the above approach to modeling the equivalent circuit of the EWH-water-anode system by its impulse response in the Simulink/Simscape software environment, the model shown in fig.7 was developed.

The model, which is shown fig. 7, includes a Signal Builder, a controlled current source (I Source 2), a model subsystem (MODEL), an ammeter (Current Sensor), a voltmeter (Voltage Sensor), Simscape signal to signal converters Simulink and an oscilloscope that displays the current and voltage graphs of the model (Scope).

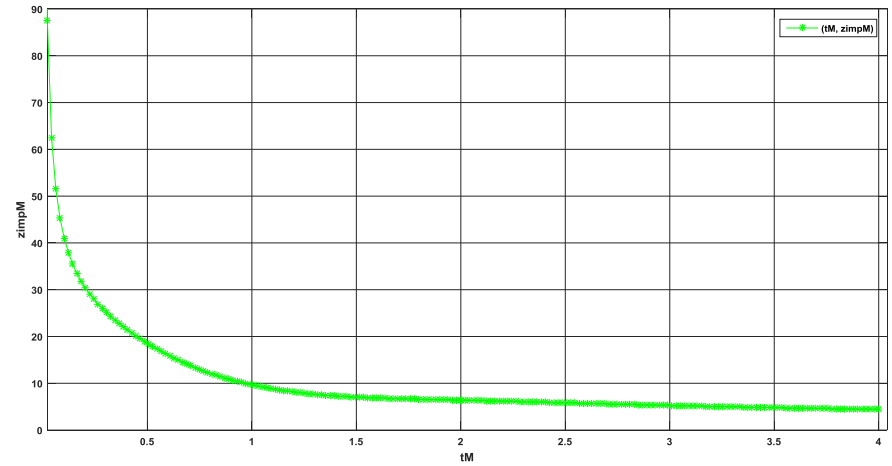

Fig. 6. The impulse response graph of the equivalent circuit of the EWH-water-anode system.

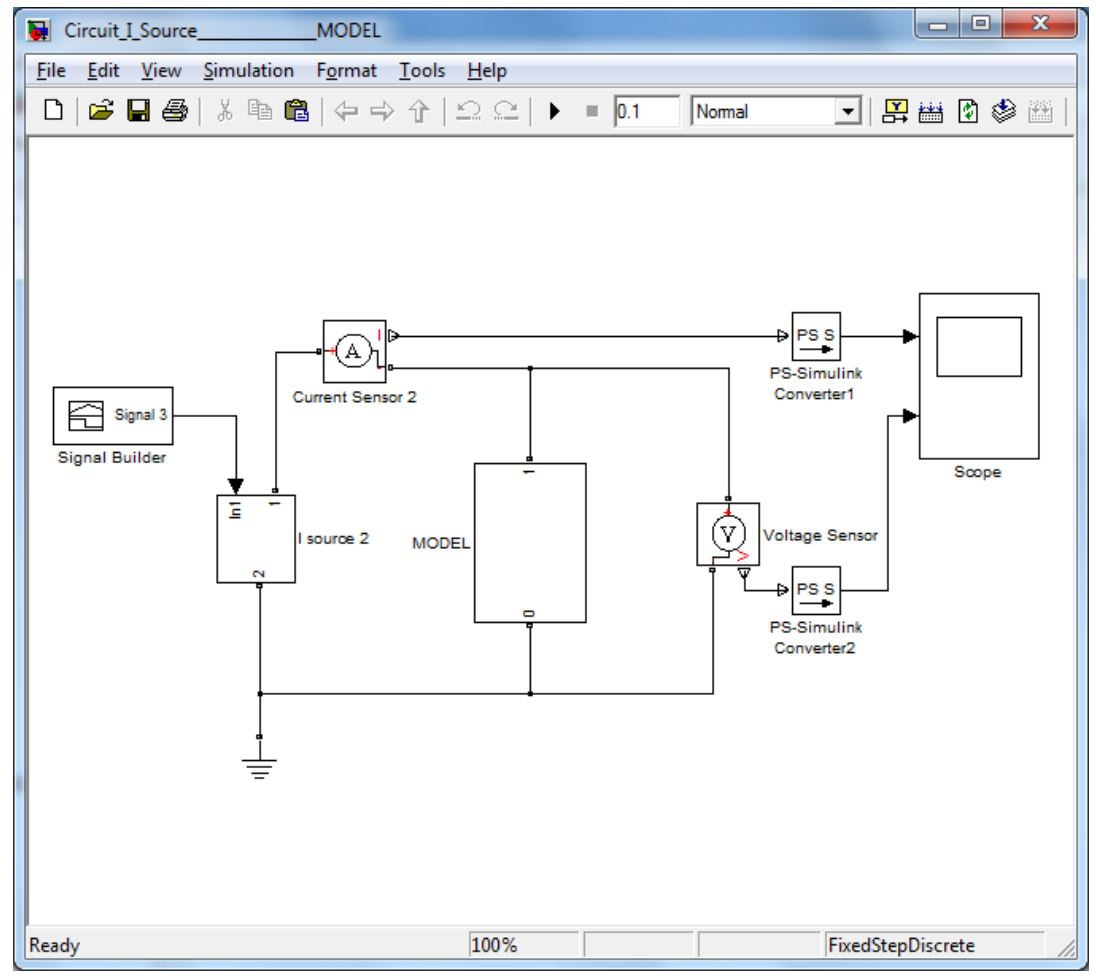

Fig. 7. Model for of equivalent circuit for the EWH-water-anode system. 


\section{Block diagram of a device for EWH corrosion protection potential diagnostics}

By analyzing the process of diagnostics of the EWH corrosion protection potential (fig. 2) and the equivalent circuit of the object (cathode) and the anode, located in a conductive medium (fig. 3), the authors propose a block diagram of a device for EWH corrosion protection potential diagnostics which is shown in fig. 8 .

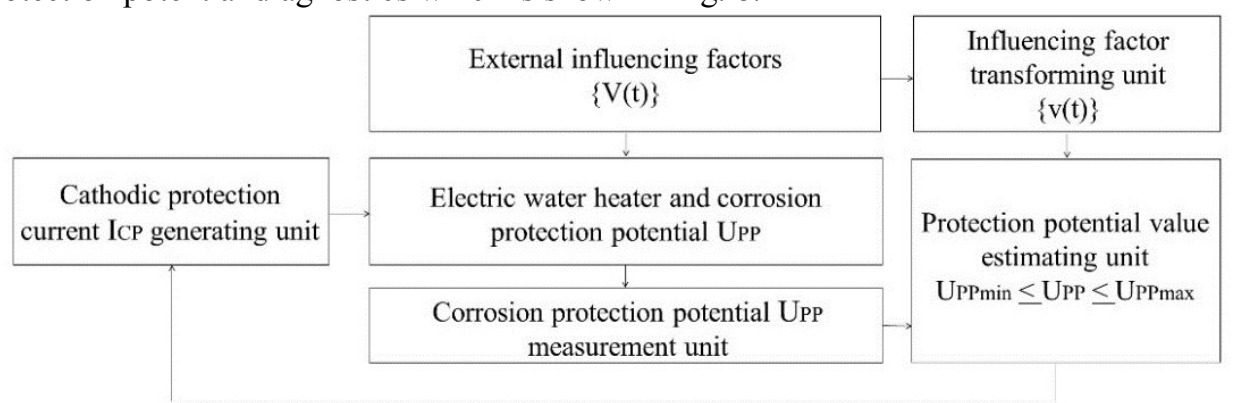

Fig. 8. General block diagram of a device for EWH corrosion protection potential diagnostics.

In the process of diagnosing the EWH corrosion protection potential, the cathodic protection current generating unit generates a cathodic protection current between the EWH body (cathode) and the anode $I_{C P}$.

In the process of the corrosion protection potential diagnostics, external influencing factors $\{\mathrm{V}(\mathrm{t})\}$ affect the EWH. The protection potential measuring unit measures $U_{P P}$, and the influencing factor transforming unit transforms them into various parameters of influencing factors $\{\mathrm{v}(\mathrm{t})\}$.

The measured [9-12] protection potential $U_{P P}$ and the variety of influencing factor parameters $\{\mathrm{v}(\mathrm{t})\}$ are sent to the protection potential value estimating unit, which determines the assessment of $\mathrm{Q}$ (whether the protection potential $U_{P P}$ falls or not into the specified tolerance $\left.U_{P P \text { min }} \leq U_{P P} \leq U_{P P \max }\right)$.

Next, two hypotheses are put forward.

1. The basic hypothesis $\mathrm{H}_{0}$ (protection potential $U_{P P}$ falls into the specified tolerance $\left.U_{P P \min } \leq U_{P P} \leq U_{P P \max }\right)$.

$$
H_{0}: U_{P P} \in\left[U_{P P \min }, U_{P P \max }\right]
$$

where: $U_{P P \min }$ and $U_{P P \max }$ are limits of permissible interval for the EWH corrosion protection potential $U_{P P}$.

2. Alternative hypothesis $\mathrm{H}_{1}$ (protection potential $U_{P P}$ does not fall into the specified tolerance $\left.U_{P P \min } \leq U_{P P} \leq U_{P P \max }\right)$.

$$
H_{1}: U_{P P} \notin\left[U_{P P \min }, U_{P P \max }\right]
$$

The instrumental diagnostic reliability of the EWH corrosion protection potential is defined by the expression, which includes the probabilities of errors of the first and second kind, $\alpha$ and $\beta$ respectively.

The probabilities of errors of the first and second kind are defined according to the expressions: 


$$
\begin{aligned}
& \alpha_{U_{P P}}=\int_{U_{P P \min }}^{U_{P P_{\max }}} f\left(U_{P P}\right)\left(\int_{-\infty}^{U_{P P \min }-U_{P P}} f\left(\delta_{U_{P P}}\right) d \delta_{U_{P P}}\right) d U_{P P}+ \\
& +\int_{U_{P P \min }}^{U_{P P \max }} f\left(U_{P P}\right)\left(\int_{U_{P P \max }-U_{P P}}^{\infty} f\left(\delta_{U_{P P}}\right) d \delta_{U_{P P}}\right) d U_{P P} \\
& \beta_{U_{P P}}=\int_{-\infty}^{U_{P P_{\min }}} f\left(U_{3 \Pi}\right)\left(\int_{U_{P P \min }-U_{3 I}}^{U_{P \max }-U_{3 \Pi}} f\left(\delta_{U_{P P}}\right) d \delta_{U_{P P}}\right) d U_{P P}+ \\
& +\int_{U_{P P_{\max }}}^{\infty} f\left(U_{P P}\right)\left(\int_{U_{P P \min }-U_{P P}}^{U_{P P_{\max }}-U_{P P}} f\left(\delta_{U_{P P}}\right) d \delta_{U_{P P}}\right) d U_{P P}
\end{aligned}
$$

where: $U_{P P_{\min }}$ and $U_{P P_{\max }}$ are limits of permissible interval for the EWH corrosion protection potential $U_{P P}$;

$f\left(U_{P P}\right)$ is density of probability for the EWH corrosion protection potential $U_{P P}$;

$f\left(\delta_{U_{P P}}\right)$ is density of measurement error probability for the EWH corrosion protection potential $U_{P P}$.

Then the general reliability of the EWH corrosion protection potential diagnostics is defined by the expression

$$
D_{G}=D_{M} \cdot D_{I}=D_{M} \cdot(1-\alpha-\beta)
$$

Assessment of the quality of the EWH corrosion protection potential diagnostics may also be calculated by the formula of the average diagnostics risk

$$
\Psi \approx W_{1 U_{P P}} \alpha+W_{2 U_{P P}} \beta
$$

where: $W_{1 U_{P P}}$ and $W_{2 U_{P P}}$ are losses due to errors of the 1st and 2nd kind.

\section{Conclusions}

By analyzing the equivalent circuit that simulates the process of diagnosing the EWH protection potential, we may conclude that traditional automatic cathodic protection devices measuring the cathodic protection potential $U_{P P}$ when the cathodic protection current $I_{C P}$ passes through, make a relative methodological error in measuring the cathodic protection potential $\delta_{M}$.

Methodological measurement error $\delta_{M}$ reduces the instrumental reliability of diagnostics of the cathode $D_{I}$ protection potential and, consequently, the general reliability of the EWH corrosion protection potential diagnostics $D_{G}$.

Methodological measurement error $\delta_{M}$ is conditioned by the voltage drops that occur due to cathodic protection current $I_{C P}$ passing through the active resistance of electrolyte, active resistance of the EWH body (cathode) and active resistance of connecting wires.

The value of the methodological measurement error $\delta_{M}$ may be described by the expression

$$
\delta_{M}=\frac{U_{C}+U_{E C}+U_{C W}}{U_{P P}}=\frac{I_{C P}\left(R_{C}+R_{E C}+R_{C W}\right)}{U_{P P}}
$$

On analyzing the expression of the methodological measurement error $\delta_{M}$, we may conclude that an increase in the cathodic protection current $I_{C P}$ and active resistances $R_{E C}$, 
$R_{C}$ and $\mathrm{R}_{\mathrm{CW}}$, leads to an increase in the methodological measurement error $\delta_{M}$ and a decrease in the instrumental and general reliability of the EWH protection potential diagnostics.

\section{References}

1. W. Baeckmann. Handbuch des kathodischen Korrosionsschutzes: Theorie und Praxis der elektrochemischen Schutzverfahren / W. Baeckmann, W. Schwenk - Weinheim: Viley-VCH, 1999 - 530 p., (1999)

2. K.J. Kim, M.H. Lee and K.M. Moon, "Principle and Application of Cathodic Protection for Concrete structures", Korea Concrete Institute (KCI) journal, (2006)

3. S. Wong, W. Muneer, S. Nazir, A. Prieur, Designing, Operating, and Simulating Electric Water Heater Populations for the Smart Grid, Report No. 2013-136 (RP-TEC), Canmet ENERGY, Varennes Research Centre, Natural Resources Canada, October (2013)

4. D. C. Montgomery and G.C. Runger, Applied Statistics and Probability for Engineers. 2nd Edition, John Wiley \& Sons, New York, (1999)

5. V. Martynyuk, O. Eromenko, J. Boiko and T. Kałaczyński. Diagnostics of supercapacitors. MATEC Web Conf., 182, 01009, (2018)

6. V. V. Martynyuk Fractional Model of an Electrochemical Capacitor / V. V. Martynyuk, M. D. Ortigueira // Journal of Signal Processing. - Vol. 107, pp. 355-360, (2015)

7. V. Martynyuk, O. Vdovin, J. Boyko and N. Vlasenko "Super-high capacitor analyzer with compensation of common-mode error," in Proc. IMECO TC-4, pp. 340-343, (2001)

8. V. Martynyuk, D. Makaryshkin, and J. Boyko, "Frequency domain analysis for electrochemical supercapacitors", in 2007 Proc. 15th IMEKO Symposium on Novelties in Electrical Measurements and Instrumentation in Parallel with the 12th Workshop on ADC Modelling and Testing (IMEKO), Iasi, pp. 5., (2007)

9. S. Galla, A. Szewczyk, J. Smulko and P. Przygocki: Methods of Assessing Degradation of Supercapacitors by Using Various Measurement Techniques // Applied Sciences-Basel. -Vol. 9, iss. 11, s.1-11, (2019)

10. V. Martynyuk, D. Makaryshkin and J. Boyko, "Electrochemical supercapacitor time domain analysis by means of multi-channel measurement system", in 2007 Proc. 15th IMEKO Symposium on Novelties in Electrical Measurements and Instrument. in Parallel with the 12th Workshop on ADC Modelling and Testing, Iasi, Vol. I, pp. 207211, (2007)

11. M. Łukasiewicz, T. Kałaczyński, J. Musiał, J. Shalapko, Diagnostics of buggy vehicle transmission gearbox technical state based on modal vibrations, Journal of Vibroengineering 16, 6, (2014)

12. . Kałaczyński, M. Łukasiewicz, J. Musiał, R. Polasik, M. Szczutkowski, N. Dluhunovych, J. Wilczarska, T. Kasprowicz. Analysis of the diagnostic potential research thermovision in the technical state of combustion engine injectors assessment, INT CONF ENG MECHS , Engineering Mechanics,(2018) 